\title{
The Influence of Electrode Geometry on Mass Sensitivity of Quartz Crystal Microbalance
}

\author{
Li Yang ${ }^{1,2, a}$, xianhe Huang ${ }^{1, b}$ \\ ${ }^{1}$ Frequency Control Laboratory, School of Automation \& Engineering, University of Electronic \\ Science and Technology of China, Chengdu, 610054, China \\ ${ }^{2}$ College of Electrical \& Information Engineering, Southwest University for Nationalities, Chengdu, \\ 610041 ,China \\ aemail: yang9707@163.com, bemail: xianhehuang@uestc.edu.cn
}

Keywords: Quartz crystal microbalance; mass sensitivity; electrode.

\begin{abstract}
The mass sensitivity of the quartz crystal microbalance is analytically calculated for various configuration and various thickness of electrode. The data indicated that both the configuration and the thickness of electrode are influence the mass sensitivity. For the conventional electrode structure, when the thickness of electrode increase from 400A to 1000A.the maxim mass sensitivity which pointed at center of electrode are increase from $0.78 \times 10^{13} \mathrm{~Hz} / \mathrm{Kg}$ to $2.41 \times$ $10^{13} \mathrm{~Hz} / \mathrm{Kg}$ and the mass sensitivity increased at same point of radius with the increase of thickness of electrode. With the decrease of the thickness of electrode, the mass sensitivity become more uniform. Analysis also show that the triple-ring electrode structure has the highest mass sensitivity compare with the ring and circle.
\end{abstract}

\section{Introduction}

The quartz crystal microbalance(QCM) has been widely used as highly sensitive sensor which commonly configure with metallic electrode on both sides of a thin disk AT-cut quartz. The crystal can be electrically excited into resonance because of the piezoelectric properties. In the late 1950s, Sauerbrey found the relationship between resonant frequency and mass deposit on surface of quartz in gas phase ${ }^{[1]}$,

$$
\Delta f=-\frac{2 f_{0}^{2}}{A\left(\mu_{q} \rho_{q}\right)^{\frac{1}{2}}} \Delta m
$$

Where $\Delta \mathrm{f}$ is the observed frequency change (in $\mathrm{Hz}$ ), $\mu_{\mathrm{q}}$ is the shear modulus, $\rho_{\mathrm{q}}$ is the density of the crystal, $\mathrm{A}$ is the active electrode area, and $\Delta \mathrm{m}$ is the mass change on the surface of the crystal (in ng). For the AT-cut quartz crystal $\left(\rho_{\mathrm{q}}=2.65 \mathrm{~g} / \mathrm{cm}^{3}, \mu_{\mathrm{q}}=2.95 \mathrm{~N} / \mathrm{m}^{2}\right)$.

For Sauerbrey equation, the deposit thin film should be rigid thin and since the work of Sauerbrey, the QCM has been widely used to monitor slight mass change(nanogram) in many research areas include biology, physics, medicine and so on ${ }^{[2-4]}$. More recently, new application of the QCM as a immunosensor for clinical diagnose has been also investigated ${ }^{[5]}$. Immunosensor are device which utilize antigen-antibody reactions for detecting target analytes . In these applications, both antigen and antibody are applied on the surface of QCM resulting in an antibody-antigen reaction, This reaction was followed by a change in the resonant frequency of QCM. It is obviously that the precision of immunosensor is related to the mass sensitivity of the QCM, so the mass sensitivity of the QCM play very important role in this study.

In this paper, the mass sensitivity of the QCM is investigated and the relationship between electrode configuration on the surface of crystal to the mass sensitivity is discussed. It is shown that, the radius and the thickness of the electrode all influence the mass sensitivity of QCM.

\section{Theory}

For the AT-cut crystal which had nearly zero frequency drift with temperature around room temperature, only the shear horizontally polarized particle displacement $\mathrm{u}$ (in the $\mathrm{X}$ direction) is 
coupled to the electrical potential. Because of the piezoelectric properties, the crystal can be excited into resonance and the change in the resonance frequency can be described as follow ${ }^{[6]}$ :

$$
\Delta \mathrm{f}=\frac{1}{\pi r_{d}^{2}} \int_{0}^{2 \pi} \int_{0}^{r_{d}} S(r, \theta) \Delta m(r, \theta) r d r d \theta
$$

In which, $S(r, \theta)$ is mass sensitivity function and represents the differential frequency change per mass change at a specific location, $\Delta \mathrm{m}(\mathrm{r}, \theta)$ is the effective added mass, $r_{d}$ is the radius for the localized or nonuniform mass deposit on the electrode, $r$ and $\theta$ are the polar coordinates of the point at which the mass is added. Mass sensitivity function could be calculated by equation(3):

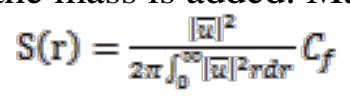

Where $\bar{u}$ is particle displacement amplitude, $\mathrm{C}_{\mathrm{f}}$ is sauerbery's sensitivity constant, $\mathrm{r}$ is the distant from center. The quartz crystal microbalance configurations are shown as figure1.

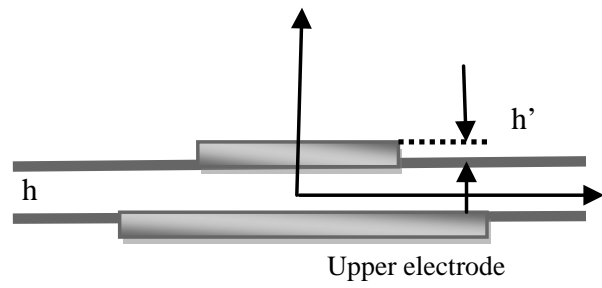

Figure1 Top view of quartz crystal microbalance

In which, the plate surface is defined as $(\mathrm{X}, \mathrm{Z})$ and the thickness of the crystal and the electrode are assumed to $\mathrm{h}$ and $\mathrm{h}$ ' respectively .The acoustic wave is generated by the electric potential applied across the electrodes. For AT-cut crystal, only the X-polarized particle displacement is coupled to the electric potential. The particle displacement $u$ can be written as equation ${ }^{[7]}(4)$

$u_{1}(x, y, z, t)=\bar{u} \sin \left(k_{2} y\right) \cos \omega t$

The surface plane of gold electrode was defined by $(\mathrm{x}, \mathrm{z}), k_{2}$ is the shear horizontal acoustic wavenumber in the $y$ direction. The equation (5) could be reduced as:

$$
\begin{aligned}
& A \frac{\partial^{2} \overline{\mathrm{u}}}{\partial x^{2}}+B \frac{\partial^{2} \overline{\mathrm{u}}}{\partial z^{2}}+k_{\mathrm{i}}^{2} \overline{\mathrm{u}}=0 \\
& \frac{\mathrm{c}_{11}}{\mathrm{c}_{66}} \frac{\partial^{2} \mathrm{u}_{1}(\mathrm{r})}{\partial \mathrm{x}_{1}^{2}}+\frac{\mathrm{c}_{55}}{\mathrm{c}_{\mathrm{Eg}}} \frac{\partial^{2} \mathrm{u}_{1}}{\partial \mathrm{x}_{3}^{2}}+\frac{\omega^{2}-\omega_{1}^{2}}{\mathrm{c}^{2}} \quad \mathrm{u}_{1}(\mathrm{r})=0 \\
& \text { In which: } \\
& A=\frac{c_{11}}{c_{66}}-2 \frac{c_{56} e_{11}}{c_{66}\left(e_{11}+e_{26}\right)} \\
& \mathrm{B}=\frac{\mathrm{c}_{55}}{\mathrm{c}_{66}}-2 \frac{\mathrm{c}_{56} e_{35}}{\mathrm{c}_{66}\left(\mathrm{e}_{25}+\mathrm{e}_{26}\right)} \\
& k_{i}^{2}=\frac{\omega^{2}-\omega_{i}^{2}}{\mathrm{c}^{2}}
\end{aligned}
$$

Where $c_{i j j}$ and $e_{i j}$ represent the elastic stiffness constant and piezoelectric constant respectively ; $\omega_{i \mathrm{i}}=k_{2} \sqrt{\frac{\sigma_{\mathrm{EE}}}{\rho}}$ is cutoff frequency, $c=\sqrt{\frac{\sigma_{\mathrm{EE}}}{\rho}}$ is the acoustic wave velocity in crystal.

Convert equation(6) into polar coordinate and considering the particle displacement amplitude has independence on $\theta$,so the equation convert as:

$\mathrm{A} \cos ^{2} \theta \frac{\theta^{2} \overline{\mathrm{u}}}{\partial \mathrm{r}^{2}}+\mathrm{A} \sin ^{2} \theta \frac{1}{r} \frac{\partial \overline{\mathrm{u}}}{\partial \mathrm{r}}+\mathrm{B} \sin ^{2} \theta \frac{\theta^{2} \overline{\mathrm{u}}}{\partial \mathrm{r}^{2}}+\mathrm{B} \cos ^{2} \theta \frac{1}{r} \frac{\partial \overline{\mathrm{u}}}{\partial \mathrm{r}}+\mathrm{k}_{\mathrm{i}}^{2} \overline{\mathrm{u}}=0$

Assume $\theta=45^{\circ}$, then

$r^{2} \frac{\partial^{2} \bar{u}}{\partial r^{2}}+\frac{\partial \bar{u}}{\partial r}+\frac{k_{i}^{2} y^{2}}{M} \bar{u}=0$

Where: $\mathrm{M}=\frac{A+B}{2}$

The equation (11) is a Bessel differential equation. The particle displacement amplitude $\bar{u}$ can be calculated, the solution for equation (7) can be written as 


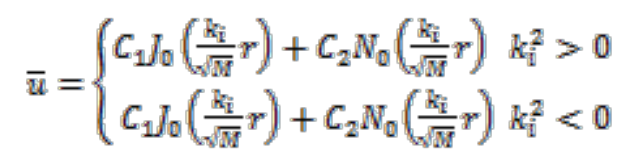

The differential equations of the forced piezoelectric thickness vibration for the AT-cut crystal are:

$$
\begin{gathered}
\mathrm{C}_{66} \frac{\theta^{2} \overline{\mathrm{u}}}{\partial \mathrm{x}_{2}^{2}}+\mathrm{e}_{26} \frac{\theta^{2} \varphi}{\partial \mathrm{x}_{2}^{2}}=\rho \frac{\theta^{2} \overline{\mathrm{u}}}{\partial \mathrm{t}^{2}} \\
e_{26} \frac{\partial^{2} \bar{u}}{\partial x_{2}^{2}}-\epsilon_{22} \frac{\partial^{2} \varphi}{\partial x_{2}^{2}}=0
\end{gathered}
$$

Where $\varepsilon_{i j}$ is the dielectric constant, ${ }^{\Phi}$ is the electrical potential applied across the electrodes.

The boundary condition for AT-cut crystal with surface electrodes in the form:

$$
c_{66} \frac{\partial^{2} \bar{u}}{\partial x_{2}^{2}}+e_{26} \frac{\partial^{2} \varphi}{\partial x_{2}^{2}} \pm 2 h^{p} \rho^{\frac{\partial^{2} \bar{u}}{\partial \tilde{r}^{2}}}=0 \text { at } x_{2}= \pm h
$$

submitted equation (4) into (13)

$$
\left(c_{66}+\frac{e_{26}^{2}}{\varepsilon_{22}}\right) k_{2}^{2}=p \omega^{2}
$$

And consider the boundary condition at the surface of electrode, for fully electrode region:

$\bar{u}\left(k_{2} h \cos k_{2} h-k_{26}^{2} \sin k_{2} h-R k_{2}^{2} h^{2} \sin k_{2} h\right)=-\frac{e_{26 \varphi_{0}}}{\overline{c_{66}}}$

Where:

$$
\mathrm{k}_{26}^{2}=\frac{\mathrm{e}_{26}^{2}}{\overline{\mathrm{C}_{66} \mathrm{z}_{22}}} \quad \mathrm{R}=\frac{2 \mathrm{p}^{\prime} \mathrm{h}^{s}}{\mathrm{ph}} \quad \overline{\mathrm{c}_{66}}=\mathrm{c}_{66}+\frac{\mathrm{e}_{2 \mathrm{E}}^{2}}{\mathrm{z}_{22}}
$$

In which ,P and $\mathrm{P}$ are density for electrode and crystal respectively.

When the coefficient of $\bar{u}$ in equation(17) vanishes, the resonance will occurs, so:

$\tan k_{2} h=\frac{k_{2} \hbar^{2}}{k_{26}^{2}+R k_{2}^{2} h^{2}}$

Solving the equation (18), the $\mathrm{k}_{2}^{\mathrm{e}}$ for the fully electrode region can be obtained as:

$k_{2}^{e}=\frac{n \pi}{2 h}\left(1-R-\frac{4 k_{26}^{2}}{\pi^{2} n^{2}}\right)$

Where, $n$ is the overtone order.

So ,the fundamental resonance frequency is:

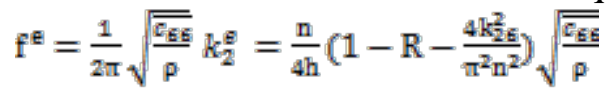

Using the same method, the fundamental resonance frequency for partially electrode region is:

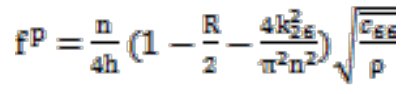

the fundamental resonance frequency for unelectrode region is:

$\mathrm{f}^{\mathrm{u}}=\frac{\mathrm{n}}{4 \mathrm{~h}}\left(1-\frac{4 \mathrm{k}_{2 \mathrm{E}}^{2}}{\mathrm{~m}^{2} \mathrm{n}^{2}}\right) \sqrt{\frac{\sigma_{\mathrm{EE}}}{\mathrm{p}}}$

Using equation(9),(20),(21),(22), the $\mathrm{k}_{\mathrm{i}}^{2}$ for different region include fully electrode, partially electrode and unelectrode can be found, then the X-polarized particle displacement amplitude also can be found through equation(12).

\section{Influence of the electrode's thickness}

The configuration of the QCM like fiugue(1),the radius of upper electrode is 3mm,the radius of lower electrode is $5 \mathrm{~mm}$,the radius of upper electrode smaller than the lower electrode.

The operating frequency for QCM is $11 \mathrm{MHz}$, the thickness of the crystal substrate for fundamental is $0.075 \mathrm{~mm}$. The thickness of the electrode change from $400 \dot{A}$ to $1000^{\AA}$, then the values of the electrode mass loading factor change from 0.0076 to 0.019 .

When $\mathrm{f}^{\mathrm{u}}>f>\mathrm{f}^{\mathrm{p}}>\mathrm{f}^{\mathrm{E}}$, for configuration like figure1, the solution of the particle displacement in each region can be obtained as: 


$$
\bar{u}=\left\{\begin{array}{cc}
C_{1} J_{0}\left(\frac{k_{e}}{\sqrt{M}} r\right) & 0 \leq \mathrm{r} \leq 3 \\
C_{2} J_{0}\left(\frac{k_{p}}{\sqrt{M}} r\right)+C_{\mathrm{a}} N_{0}\left(\frac{k_{p}}{\sqrt{M}} r\right) & 3 \leq \mathrm{r} \leq 5 \\
C_{4} K_{0}\left(\frac{k_{u}}{\sqrt{M}} r\right) & 5 \leq \mathrm{r} \leq \infty
\end{array}\right.
$$

The amplitude constant $C_{1}, C_{2}, C_{a}, C_{4}$ are determined from the boundary condition. The boundary condition is the particle displacement $\bar{u}$ and the $\frac{\partial \bar{u}}{\partial r}$ are continuity at $r=3,5$.

Figure 2 compares the differential mass sensitivity profiles of various value of electrode mass loading factor $\mathrm{R}$ for a given type of QCM.

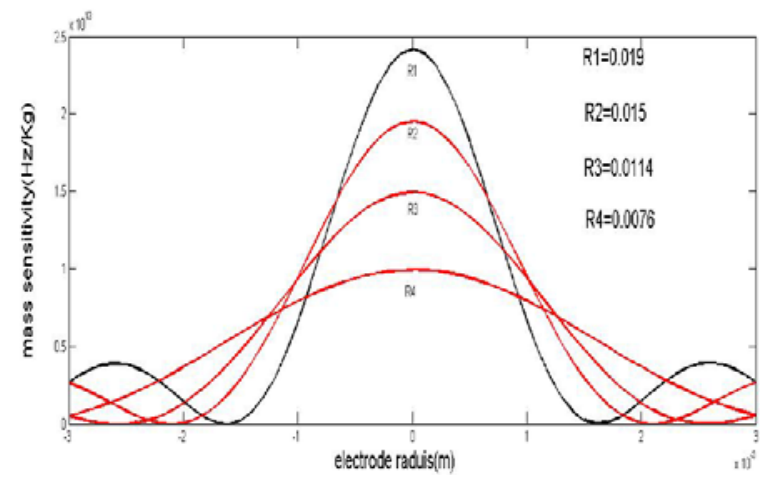

Figure2 Mass sensitivity for different electrode mass loading factor

The figure2 shown that the profile of mass sensitivity for QCM Along the radial direction which were obtained with the same electrode geometry (like figure1) and different mass loading factor(change from 0.0075 to 0.019 ). we can see from figure2 that the mass sensitivity was decrease along the radial direction and they have the Gaussian shape. All the curves in the figure2 have the higher mass sensitivity point at the center of electrode. With the increase of electrode mass loading factor(R) the mass sensitivity were increase at the same point of electrode. It is obviously that with the increase of the electrode mass loading factor the mass sensitivity for QCM were increased and the increase of the electrode mass loading factor can obtained by increased the thickness of electrode. It is also can observed that the curve of figure2 become more uniformity with the decrease of electrode mass loading, but the uniform of the mass sensitivity of QCM sacrifice some absolute mass sensitivity.

\section{Influence of the electrode configuration}

Three type structure of electrode for QCM were selected in this paper. They are triple-ring electrode structure, ring electrode structure and

The fundamental operating frequency for all the three type QCM are $11 \mathrm{MHz}$, the thickness of the crystal substrate for fundamental operating frequency $11 \mathrm{MHz}$ is $0.075 \mathrm{~mm}$ and thickness of the electrode for all three type structure are set as $800 \dot{A}$. For the triple-ring electrode(figure3),the parameters are selected as $\mathrm{s}=1 \mathrm{~mm}, \mathrm{t}=2 \mathrm{~mm}, \mathrm{k}=3 \mathrm{~mm}, \mathrm{~m}=4 \mathrm{~mm}, \mathrm{n}=5 \mathrm{~mm}$. for the ring-electrode ,the parameter are selected as $g=1.5 \mathrm{~mm}, \mathrm{~h}=3.5 \mathrm{~mm}$. for the circle electrode $\mathrm{j}=5 \mathrm{~mm}$.

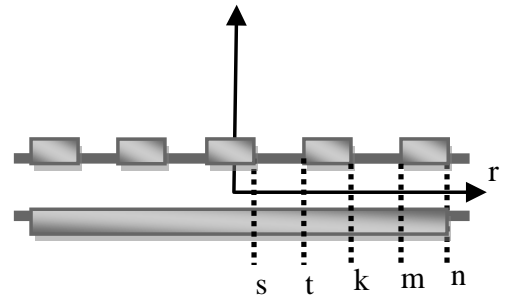

(a)

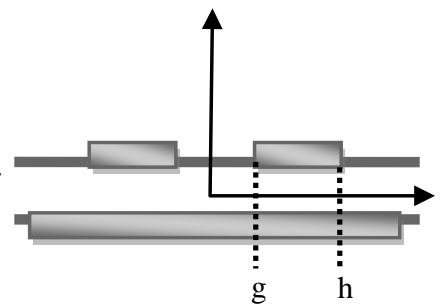

(b)

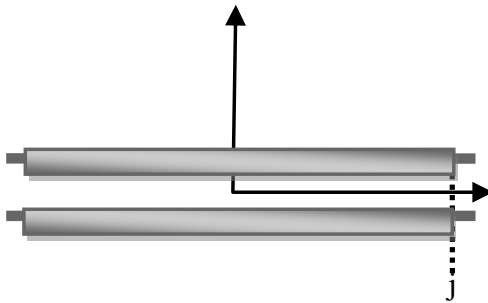

(c)

Figure3 Top view of three type of the electrode configuration.(a) triple-ring electrode.(b)ring electrode. 
(c)circle electrode, where the diameter of the upper electrode is equal to the lower electrode diameter. the profile of mass sensitivity distribution for these three type of QCM with various electrode configuration can be obtained as shown in Figure4.

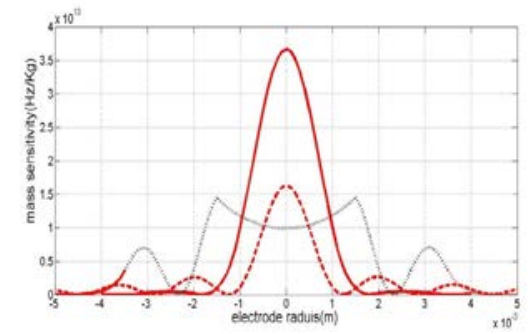

Figure4 Mass sensitivity distribution for ring ,triple-ring and circle electrode quartz crystal microbalance

It is very clearly to shown that the profile of mass sensitivity distribution have Gaussian shape for triple-ring and circle electrode. The maxim value of mass sensitivity are $3.66 \times 10^{13}$, $1.63 \times 10^{13}$ for triple-ring and circle electrode respectively. The higher mass sensitivity are all located at center of electrode for triple-ring and circle electrode structure. For ring electrode structure, the profile of the mass sensitivit more uniform but sacrifice some absolute mass sensitivity.

\section{Conclusion}

In this work, the geometry of electrode influence on the mass sensitivity of quartz crystal microbalance with fundamental operating frequency of $11 \mathrm{MHz}$ is discussed. With the decrease of the thickness of electrode, the mass sensitivity decreased at the same point of electrode but on the other hand the profile of mass sensitivity become more uniform. Analysis also show that the triple-ring electrode structure has the highest mass sensitivity compare with the ring and circle electrode structure.

\section{REFERENCES}

[1]Sauerbrey,G. "Use of quartz vibrator for weighting thin film on a microbalance" Z.phys.vol.155,pp206-212, 1958.

[2] Kanazawa, K and Gordon, J. G. "The oscillation frequency of a quartz resonator in contact with liquid”. Anal. Chim. Acta, vol.175, pp99-105, 1985

[3] Sahebali S, Depuydt CE, Boulet GAV, et al. "Immunocytochemistry in liquid-based cervical cytology: analysis of clinical use following a cross-sectional study. Int $\mathrm{J}$ Cancer.; vol.118,pp1254-1260,2006.

[4] J. Ferlay, H.R. Shin, F. Bray, Estimates of worldwide burden of cancer in 2008: GLOBOCAN. Int. J. Cancer .127(2008)2893-2917.

[5] Li Yang, Xianhe Huang, A Piezoelectric Immunsensor for Early Cervical Cancer Detection Asian Pac J Cancer Prev, ,9375-9377,2014.

[6] A. C. Hillier and M. D. Ward. "Scanning Electrochemical Mass Sensitivity Mapping of the Quartz Crystal Microbalance in Liquid Media”. Anal. Chem.vol.64,pp2539-2554, 1992,

[7] Tiersten, H. F.Linear Piezoelectric Plate Vibration; Plenum Press: New York,1969,pp102-121 